Apidologie, 1987, 18 (4), 327-334

RAPID COMMUNICATION

\title{
CONSTITUENTS OF PROPOLIS
}

\author{
Penelope Walker and Eva Crane \\ Woodside House, Woodside Hill, Gerrards Cross, Bucks SL98NX, UK
}

\begin{abstract}
SUMMARY
Constituents of propolis reliably reported in the literature are listed. Flavonoids (38) form the largest group of the total number (149), and there are 14 derivatives of cinnamic acid and 12 of benzoic acid. Eleven other groups include terpene and sesquiterpene alcohols, and hydrocarbons.
\end{abstract}

There is currently much interest in the propolis used by honeybees in their nest or hive, and in its pharmacological properties (e.g. KönIG and DustmanN, 1986). The sticky plant substances that are collected by bees may include different types of secretion (lipophilic substance, mucilage, gum, oil, possibly wax), or exudate (resin, latex) (FAHN, 1986). These substances have no general botanical name, and the same word "propolis» is used for them as for the material in the hive. It is considered unlikely that honeybees alter the composition of propolis after they collect it (MCGREGOR, 1952; KöNIG, 1985).

It seems important that up-to-date information on the plant origins and composition of propolis should be made available. Types of reference material previously lacking in the literature include : $(a)$ information on the plants from which bees collect propolis in different countries, and the type of material collected ; $(b)$ a complete list of compounds so far identified in propolis. Of these, (a) will be dealt with elsewhere (CRANE, 1988), in a Table giving plant sources world-wide and indicating the type of secretion or exudate that constitutes the propolis from them. A list of compounds identified in propolis (b) is published here. 
TABL. 1. - Compounds identified in propolis

References (coded e.g. G79) are listed at the end of the paper.

\section{Flavonoids}

G79 lists the following :

\section{Chrysin}

Tectochrysin

Acacetin

\section{Quercetin}

Kaempferide

Rhamnocitrin

\section{Galangin}

Isalpinin

Ermanin

Pectolinarigenin

Pinostrobin

Pinocembrin

Sakuranetin

Isosakurinetin

Quercetin-3,3'-dimethyl ether

Pinobanksin

3-acetyl pinobanksin 5,7-dihydroxyflavone

5-hydroxy-7-methoxyflavone

5,7-dihydroxy-4'-methoxyflavone

5-hydroxy-4', 7-dimethoxyflavone

$3,3^{\prime}, 4^{\prime}, 5,7$-pentahydroxyflavone

$3,5,7$-trihydroxy-4'-methoxyflavone

3,4',5-trihydroxy-7-methoxyflavone

3,5-dihydroxy-4',7-dimethoxyflavone

3,5-7-trihydroxyflavone

3,5-dihydroxy-7-methoxyflavone

5,7-dihydroxy-3,4'-dimethoxyflavone

5,7-dihydroxy-4',6-dimethoxyflavone,

5-hydroxy-7-methoxyflavanone

5-hydroxy-4',7-dimethoxyflavanone

5,7-dihydroxyflavanone

4',5-dihydroxy-7-methoxyflavanone

5,7-dihydroxy-4'-methoxyflavanone

4',5,7-trihydroxy-3,3'-dimethoxyflavone

3,5,7-trihydroxyflavanone

5,7-dihydroxy-3-acetylflavanone

Other publications list the following :

Betuletol

Isorhamnetin

Kaempferol

Apigenin

Rhamnazin

Rhamnetin

Alnusin

E

Alpinetin

-

Alnusitol 3,5,7-trihydroxy-4',6-dimethoxyflavone

V79a

$\mathrm{V} 79 \mathrm{a}$

3,4',5,7-tetrahydroxyflavone V79a

4',5,7-trihydroxyflavone

V79a

V79a

V79b

Mo85

P76

P76

P76

P76

Ko87

Ko87

Mo85

Mo85

B83

B83

Mo85

\section{Chalcones}

2,6-dihydroxy-4-methoxychalcone 
TABL. 1. - (cont.)

\section{Benzoic acid and derivatives}

Benzoic acid

Salicylic acid (2-hydroxybenzoic acid)

V79b

4-hydroxybenzoic acid

V79a

4-methoxybenzoic acid

v79a

2-amino-3-methoxybenzoic acid

M84

Gentisic acid (2,5-dihydroxybenzoic acid)

V79b

Protocatechuic acid (3,4-dihydroxybenzoic acid)

v79a

Gallic acid (3,4,5-trihydroxybenzoic acid)

V79a

Phenylmethyl ester of benzoic acid

M83

$\begin{array}{ll}\text { Phenylmethyl ester of salicylic acid } & \text { M83 }\end{array}$

$\begin{array}{ll}\text { Trans-p-coumaryl benzoate } & \text { P84 }\end{array}$

$\begin{array}{lr}\text { Trans-coniferyl benzoate } & \text { P84 }\end{array}$

\section{Benzaldehyde derivatives}

$\begin{array}{ll}\text { Vanillin } & \text { G79 }\end{array}$

$\begin{array}{ll}\text { Isovanillin } & \text { G79 }\end{array}$

5. Cinnamyl alcohol, and cinnamic acid and its derivatives

$\begin{array}{ll}\text { Cinnamyl alcohol } & \text { G79 }\end{array}$

$\begin{array}{ll}\text { Cinnamic acid } & \text { G79 }\end{array}$

3,4-dimethoxycinnamic acid $\quad$ M84

$\begin{array}{ll}o \text {-coumaric acid } & \mathrm{V79b}\end{array}$

$p$-coumaric acid (also its benzyl ester) $\quad$ V79a

$\begin{array}{ll}\text { Caffeic acid } & \text { G79 }\end{array}$

$\begin{array}{ll}\text { Hydrocaffeic acid } & \text { V79b }\end{array}$

$\begin{array}{ll}\text { Ferulic acid } & \text { G79 }\end{array}$

$\begin{array}{ll}\text { Isoferulic acid } & \text { V79a }\end{array}$

$\begin{array}{ll}\text { Cinnamic acid methyl ester } & \text { M84 }\end{array}$

Cinnamic acid ethyl ester $\quad$ M83

$\begin{array}{ll}\text { 1,3-diferuloyl-2-acetylglycerol } & \text { P82 }\end{array}$

$\begin{array}{ll}\text { 1-feruloyl-3p-coumaroyl-2-acetylglycerol } & \text { P82 }\end{array}$

$\begin{array}{ll}\text { Cinnamylidene acetic acid } & \text { Mo85 }\end{array}$

6. Other acids and derivatives

Methyl ester of 2,8-dimethylundecanoic acid $\quad$ M83

Phenylmethyl ester of 14-methylpentadecanoic acid $\quad$ M83

$\begin{array}{ll}\text { Ethyl ester of palmitic acid (hexadecanoic acid) } & \text { M83 }\end{array}$

$\begin{array}{ll}\text { Myristic acid (tetradecanoic acid) } & \text { G79 }\end{array}$

$\begin{array}{ll}\text { Sorbic acid (2,4-hexadienoic acid) } & \text { G79 }\end{array}$

$\begin{array}{ll}\text { Butyl-2-methylpropyl ester of phthalic acid } & \text { M83 }\end{array}$

$\begin{array}{ll}\text { Stearic acid } & \text { M84 }\end{array}$

$\begin{array}{lr}\text { Methyl ester of alnustic acid } & \text { Mo85 }\end{array}$ 
TABL. 1. - (cont.)

7. Alcohols, ketones, phenols and heteroaromatic compounds

Benzyl alcohol

3,5-dimethoxybenzyl alcohol

1,5-pentanediol monobenzoate

6,10,14-trimethyl-2-pentadecanone

M83

2-heptadecanone

M83

4-allyl-2-methoxyphenol

G79

Hexadecanol acetate

M83

Pterostilbene

G79

Xanthorrhoeol

G79

Coumarin

$\mathrm{V79b}$

Scopoletol

V79b

3,5-dihydroxystilbene (pinosylvin)

Mo85

8. Terpene and sesquiterpene alcohols and their derivatives

Geraniol

Nerolidol

M83

Guaiol

M83

Farnesol

M83

Dihydroeudesmol

M83

$\beta$-bisabolol

V79a

$\alpha$-acetoxybetulenol

P76

9. Sesquiterpene and triterpene hydrocarbons

$\beta$-bourbonene

M83

Caryophyllene

M83

Patchoulane

M83

Selenene

M83

Aromadendrene

M83

Calarene

M83

Copaene

M83

Calamenene

M83

$\beta$-patchoulene

M83

$\beta$-bisabolene

M83

Squalene

Kh81

10. Aliphatic hydrocarbons

Eicosane

Eicosine

Heneicosane

1-octadecene

11. Minerals

$\mathrm{Na}, \mathrm{K}, \mathrm{Mg}, \mathrm{Ca}, \mathrm{Ba}, \mathrm{Sr}, \mathrm{Zn}, \mathrm{Cd}, \mathrm{Al}, \mathrm{Si}, \mathrm{Sn}, \mathrm{Pb}, \mathrm{Fe}, \mathrm{Ni}, \mathrm{Cr}, \mathrm{Mn}, \mathrm{Ti}$ 
TABL. 1. - (cont.)

12. Sterols and steroid hydrocarbons

Cholestrilene

M82

Cholinasterol

M82

Stigmasterol

M82

$\beta$-dihydrofucosterol

M82

Lanosterol

Kh81

Cholesterol

Kh81

13. Sugars

d-ribofuranose

M84

d-fructose

M84

d-glucitol

M84

d-gulose

M84

Talose

M84

Sucrose

M84

d-glucose

M84

14. Amino acids

16 determined quantitatively

K80

24 determined quantitatively

Ga86

Table 1 includes 149 compounds and 22 minerals so far identified in propolis and reported in the literature, chemically similar compounds being grouped together. Deblock-Bostyn (1982) mentions a few other constituents, without references. GhisalberTI's literature review of propolis (1979) discusses its composition, and lists 20 flavonoids, which are an important group of components. (The paper states that a number of vitamins have been found in propolis, but the reports cited deal only with royal jelly and bee bread).

In Table 1 the publication quoted for each compound is usually the latest reliable one we have found, and is not necessarily the original report of the presence of the compound in propolis. Individual compounds were identified in analyses of one or more different samples, which originated from one or a number of plant species. Some compounds are probably present in all samples of propolis and contribute to its characteristic properties. Some may be present in many samples of different origins, but others almost certainly occur only in propolis collected from a particular plant species.

Many studies on the properties and composition of propolis have been made without knowledge of the plant(s) from which the sample came, or of the site on the plant from which the bees collected the material (which might 
help to indicate the type of plant secretion involved). In contrast, studies on the composition of nectar or pollen normally state the plant source. It is, however, very much easier to observe bees collecting nectar or pollen than those collecting propolis - often high up in trees.

Received for publication in July 1987. Accepted for publication in October 1987.

\section{ACKNOWLEDGEMENTS}

We much appreciate the assistance we have received from $M$. and $R$. VANHAELEN, V. GHISAlberti, and especially from Dr. W. MACIEJEwicz who provided several recent references which added a number of compounds to our list.

\section{RÉSUMÉ \\ LES CONSTITUANTS DE LA PROPOLIS}

Un grand intérêt se manifeste actuellement pour la propolis : pour son utilisation par les abeilles dans la ruche et pour ses propriétés pharmacologiques (cf. KöNIG et DustmanN, 1986). Les substances poisscuses récoltées par les abeilles comprennent divers types de sécrétions (substances lipophiles et mucilagincuses, gomme, huile et peut-ĉtre cire) ou d'exsudats (résine, latex) (FAHN, 1986). Aucun terme botanique général ne désigne ces substances et le terme "propolis » est utilisé aussi bien pour elles que pour le matériau présent dans la ruche. Il semble peu probable que les abeilles modifient la composition de la propolis après l’avoir récoltće (MCGregor, 1952 : KöNIG, 1985).

Il semble important de résumer les connaissances actuelles sur l'origine botanique et la composition de la propolis. Le type d'informations absent jusqu'à présent dans la littérature comprenait : a) des informations concernant les plantes sur lesquelles les abeilles récoltent la propolis dans les différents pays et le type de matériau récolté ; b) une liste complète des substances identifiées à ce jour dans la propolis. Le point a) sera traité ailleurs (CRANE, 1988) dans un tableau répertoriant les plantes du monde entier fournissant de la propolis, avec indication du type de sécrétion ou d'exsudat qui la constitue. Nous publions ici une liste des composés identifiés dans la propolis (b).

Le tableau 1 comprend 149 composés et 22 minéraux identifiés jusqu'à présent et mentionnés dans la littérature ; ils sont regroupés en fonction de leur composition chimique. La publication citée dans le tableau 1 pour chaque composé est généralement la référence fiable la plus récente que nous ayons trouvée et non pas nécessairement le travail qui a mentionné la première fois la présence du composé en question. Les composés ont été identifiés par des analyses portant sur un ou plusieurs échantillons d'une ou de plusieurs espèces de plantes. Certains composés sont probablement présents dans tous les échantillons de propolis, lui conférant ses propriétés caractéristiques.

De nombreuses études sur les propriétés et la composition de la propolis ont été faites sans connaître l'origine botanique ni géographique de l'échantillon de propolis. Par contre les études sur le pollen ou le nectar précisent habituellement l'origine botanique. Mais il est bien sûr beaucoup plus facile d'observer des abeilles, récolter du nectar ou du pollen que de la propolis - souvent haut dans les arbres. 


\section{ZUSAMMENFASSUNG \\ DIE BESTANDTEILE VON PROPOLIS}

Gegenwärtig besteht großes Interesse an der Propolis, wie es von den Bienen in ihrem Nest oder Stock verwendet wird, vor allem hinsichtlich seiner pharmakologischen Eigenschaften (z.B. KöNIG und Dustmann, 1986). Die klebrigen Substanzen pflanzlicher Herkunft, die von den Bienen gesammelt werden, umfassen Absonderungen verschiedener Art (fettlösliche und schleimartige Stoffe, Gummi, Öl, möglicherweise Wachs) oder Exsudate (Ausflüsse) wie Harz und Latex (FAHN, 1986). Die Substanzen haben keine allgemeine botanische Bezeichnung ; dasselbe Wort «Propolis » wird sowohl für sie wie für das Material im Bienenstock benutzt. Es wird für unwahrscheinlich gehalten, daß dic Bienen dic Zusammensetzung der Propolis nach dem Sammeln verändern (McGregor, 1952 ; KöNIG, 1985).

Es erscheint wichtig, den gegenwärtigen Wissensstand über die pflanzliche Herkunft und die Zusammensetzung der Propolis zusammenzufassen. Die Unterlagen, die in der Literatur bisher nicht ohne weiteres zugänglich waren, umfassen : a) Informationen über die Pflanzen, von denen die Bienen in verschiedenen Ländern Propolis sammeln, und die Art des gesammelten Materials ; b) eine vollständige Liste der Stoffe, die bisher im Propolis bestimmt worden sind. Punkt a) wird an anderer Stelle behandelt (Crane, 1988), wo in einer Tabelle weltweit pflanzliche Quellen mit Angabe der Art der Propolis (Sekret oder Exsudat) angegeben sind. Hier wird eine Liste der bisher im Propolis bestimmten Stoffe veröffentlicht.

Tabelle 1 umfaßt 149 chemische Verbindungen und 22 Minerale, die in der Literatur aufgeführt sind ; chemisch verwandte Stoffe sind zu Gruppen zusammengefaßt. Die in Tab. 1 für jede Verbindung angeführten Publikationen sind gewöhnlich dic jüngsten zuverlassigen die wir finden konnten und nicht notwendigerweise die ersten Angaben über das Vorkommen dieses Stoffes in Propolis. Einzelne Stoffe wurden bei Analysen von nur einer oder von mehreren Proben gefunden, die von einer oder einer Anzahl Pflanzenarten stammten. Einige Verbindungen sind aber wahrscheinlich in allen Propolis-Proben vorhanden und für dessen charakteristischen Eigenschaften verantwortlich.

Viele Untersuchungen über Eigenart und Zusammensetzung von Propolis wurden durchgeführt ohne dic Pflanzenart zu kennen, oder die Stelle an der Pflanze, von der die Bienen das Material gesammelt hatten. Im Gegensatz dazu wird bei Untersuchungen über Nektar oder Pollen gewöhnlich die pflanzliche Herkunft angegeben. Es ist aber viel einfacher, Bienen bei Sammeln von Nektar oder Pollen als beim Sammeln von Propolis zu beobachten - oftmals hoch oben in Bäumen.

\section{REFERENCES}

Code letters refer to Table 1.

B83 Bankova V.S., Popov S.S., Marekov N.L., 1983. - A study of flavonoids of propolis. J. Natur. Prod., 46 (4), 771-774.

CRANE E., 1988. - Beekeeping : science, practice and world resources. Heinemann, London, to be published.

Deblock-Bostyn G., 1982. - L'abeille et ses produits. Bull. Soc. Pharm. Lille, 38 (3/4), 181-203.

FAHN A., 1986. - Personal communication.

G79 Ghisalberti E.L., 1979. - Propolis : a review. Bee Wld, 60 (2), 59-84.

Ga86 Gabrys J., et al., 1986. - Free amino acids in bee hive product (propolis) as identified and quantified by gas-liquid chromatography. Pharm. Res. Comm., 18 (6), 513-518.

K80 Kardakov V.P., 1980. - [Propolis, amino acids and European foul brood.] Tekhnol. Proizvod. Prod. Pchelovod., 151-153. 
Kh81 Kholodova Y.D., et al., 1981. - [Squalene, lanosterol and cholesterol of propolis and its probable sources.] Khim. Biol. Nauki (5), 88-90.

KöNIG B., 1985. - Plant sources of propolis. Bee Wld, 66 (4), 136-139.

König B., Dustmann J.H., 1986. - Propolis und Viren : der gegenwärtige Forschungsstand. Apidologie, 17 (4), 334-336.

Ko87 KöNIG B., 1987. - Antiviral drugs from propolis. Bee Wld, to be published.

M82 MACIEJEwicz W., et al., 1982. - Gas chromatography - mass spectrometry investigation of propolis. Analysis of $\beta$-steroids. Acta Polon. Pharm., 39 (4), 277-279.

M83 Maciejewicz W., et al., 1983. - Gas chromatography - mass spectrometry investigation of propolis. Analysis of sesquiterpenes. Acta Polon. Pharm., 40 (2), 251-253.

M84 Maciejewicz W., Daniewski M., Mielniczuk Z., 1984. - Gas chromatography - mass spectrometry investigation of propolis. Analysis of phenolic acids and sugars. Chemia Analit. (Warsaw), 29 (2), 421-427.

M85 Maciejewicz W., 1985. - Chemical composition of propolis : latest investigation and own research. International Symposium on Apitherapy, Cracow, Poland, 23-25 May 1985.

MCGregor S.E., 1952. - Collection and utilization of propolis and pollen by caged honey bee colonies. Am. Bee J., 92 (1), 20-21.

Ma79 Marinescu I., Tamas M., 1979-1980. - [Phytochemical analysis of poplar buds, a possible source of propolis]. Apicultura in Romania, 54 (12), 14-16 ; 55 (2), 21-22.

Mo85 Mochida S., Haga M., Takino Y., 1985. - Chemical constituents and anti-microbial activity of Japanese propolis. Proc. 30 int. Beekeeping Congr., 455-456.

P76 Popravko S.A., 1976. - [Plant sources of propolis.] Pchelovodstvo, 96 (7), 38-41.

P82 Popravko C.A., Sokolov I.V., Torgov I.V., 1982. - [New natural phenolic triglycerides.] Khim. prir. Soedin. (2), 169-173.

P84 Popravko C.A., Sokolov I.V., Torgov I.V., 1984. - [Derivatives of unsaturated aromatic alcohols in propolis and gum benzoin.] Khim. prir. Soedin., (1), 152-160.

S77 Scheller S., et al., 1977. - Biological properties and clinical application of propolis. I. Some physico-chemical properties of propolis. Arzneimittel-Forsch., 27 (4), 889-890.

V79a Vanhaelen M., Vanhaelen-Fastré R., 1979. - Propolis I. Origine micrographie, composition chimique et activité thérapeutique. J. Pharm. Belg., 34 (5), 253-259.

V79b Vanhaelen M., Vanhaelen-Fastré R., 1979. - Propolis II. Identification par chromatographies haute-performance (liquide, gaz-liquide et sur couches minces) des constituants. Ibid., 34 (6), 317 328.

Note added in proof :

E. Wollenweber et al. (1987) have reported 7 further flavonoids in propolis. Z. Naturforsch., 42C, 10301034. 